\title{
ALGUNOS PUNTOS CLAVE SOBRE LA RADIOFRECUENCIA
}

\section{SOME KEY POINTS ON RADIOFREQUENCY}

Marcos ALMIRÓN ${ }^{1}$.

${ }^{1}$ Kinesiólogo residente, Facultad de Ciencias Médicas, Universidad Nacional de Asunción, San Lorenzo - Paraguay.

Cómo citar este artículo: Almirón M. Algunos puntos clave sobre la radiofrecuencia. Medicina Clínica y Social. 2018;2(1):41-44.

\section{RESUMEN}

La suposición de Arséne D'Arsonval, en el siglo de XIX, de que el paso de la corriente eléctrica de alta frecuencia era un potencial agente terapéutico dio paso a que otros investigadores (como Zeynek y Nagelschmidt) demostraran que el principal agente terapéutico al aplicar corrientes de alta frecuencia al organismo era el aumento de temperatura en profundidad. En la actualidad, la radiofrecuencia se considera un tipo de onda o radiación electromagnética con alta frecuencia que se encuentra en la categoría de radiaciones no ionizantes y cuyo uso está extendido en la Medicina y en la Kinesiología y Fisioterapia. En esta pequeña puesta al día, se hace un resumen de sus modalidades, de la técnica de su aplicación y de sus efectos fisiológicos en el organismo.

Palabras clave: Corriente eléctrica; Radiofrecuencia; Kinesiología; Fisioterapia.

\section{ABSTRACT}

Arséne D'Arsonval's assumption, in the 19th century, that the passage of high frequency electric current was a potential therapeutic agent gave way to other researchers (such as Zeynek and Nagelschmidt) demonstrating that the main therapeutic agent when applying high frequency currents to the organism was the increase of temperature in depth. At present, radiofrequency is considered a type of wave or electromagnetic radiation with high frequency that is in the category of non-ionizing radiation and whose use is widespread in Medicine and in Kinesiology and Physiotherapy. In this brief update, a summary is made of its modalities, the technique of its application and its physiological effects in the organism.

Keywords: Electric current; Radiofrequency, Kinesiology; Physiotherapy.

\section{RESEÑA HISTÓRICA}

En el siglo XIX, Arséne D'Arsonval demostró que el riesgo de electrocución al someterse al paso de una corriente eléctrica disminuía conforme aumentaba la frecuencia. También observó que el tejido al ser atravesado por corrientes de alta frecuencia se calentaba. D'Arsonval suponía que el paso de la corriente eléctrica de alta frecuencia era el principal agente terapéutico y consideraba el efecto térmico como un efecto colateral sin mayor significado. Con el paso del tiempo investigadores como Zeynek y Nagelschmidt demostraron que el principal agente terapéutico al aplicar corrientes de alta frecuencia al organismo era precisamente el aumento de temperatura en profundidad, por ello la técnica pasó a llamarse en su tiempo 
endotermia y posteriormente diatermia en sus dos modalidades capacitiva y resistiva. Por su parte, Nicola tesla por medio de sus trabajos sobre las corrientes de alta frecuencia puso a disposición de investigadores posteriores los medios para generar este tipo de corrientes, lo que ha generado que la alta frecuencia diatérmica se convierta en la actualidad en el estándar para la aplicación de calor profundo en el cuerpo humano (1).

\section{DEFINICIÓN}

El término radiofrecuencia (RF) hace referencia a la porción menos energética del espectro electromagnético. Es un tipo de onda o radiación electromagnética con alta frecuencia (400$500 \mathrm{~Hz}$ ) que se encuentra en la categoría de radiaciones no ionizantes. Sus primeras aplicaciones con fines médicos datan de 1931, cuando Kirschner la usó por primera vez para tratar una neuralgia del trigémino (2).

\section{MODALIDADES DE RADIOFRECUENCIA Y SUS CARACTERÍSTICAS}

Las radiofrecuencias empleadas en la actualidad pueden ser "ablativas" (para uso médico en dolor, cáncer y como electrobisturí) y "no ablativas" (para uso kinésico). La tabla 1 resume la clasificación de aquellas modalidades no ablativas (3).

TABLA 1. CLASIFICACIÓN DE LAS RADIOFRECUENCIAS NO ABLATIVAS

\begin{tabular}{|c|c|}
\hline Tipo & Características \\
\hline Inductivas & $\begin{array}{l}\text { - Monopolares: un aplicador, son las más fáciles de encontrar. } \\
\text { Bipolares: un aplicador (o electrodo activo) y una placa disper- } \\
\text { siva. }\end{array}$ \\
\hline Capacitivas & $\begin{array}{l}\text { - Monopolares: un solo electrodo. Raras de encontrar. } \\
\text { - } \quad \text { Bipolares: dos electrodos. }\end{array}$ \\
\hline Resistivas & $\begin{array}{l}\text { - Monopolares: un solo electrodo. Raras de encontrar. } \\
\text { - Bipolares: dos electrodos. }\end{array}$ \\
\hline
\end{tabular}

En los equipos bipolares suelen haber electrodos dispersos o pasivos (de mayor área) y electrodos activos (más pequeños). El incremento de temperatura sucederá debajo del electrodo activo ya que la radiación electromagnética estará concentrada en una menor superficie. Además, los electrodos pudieran estar separados (coplanar/contraplanar) o estar en un solo cabezal (coplanar) (3).

Además de los distintos sistemas de radiofrecuencia hay diferentes formas de aplicarla. La RFp se administra mediante pulsos breves de RF de alto voltaje, pudiendo alcanzar temperaturas en torno a $40-42^{\circ} \mathrm{C}$, con disipación del calor entre pulsos evitando la coagulación del tejido. El tipo de efecto que presenta en el tejido es por tanto poco destructivo presentando actividad principalmente neuromoduladora, aunque su mecanismo de acción es aún desconocido. La 
RFc, también llamada térmica o convencional, se administra como su propio nombre indica de forma continua, sin periodos en los que se disipe el calor y con temperaturas de hasta 80$82^{\circ} \mathrm{C}$. Su efecto biológico consiste en la destrucción del tejido sobre el que se aplica, ya que a partir de $50-55^{\circ} \mathrm{C}$ el daño celular es irreversible $(4,5)$.

\section{TÉCNICA DE APLICACIÓN Y DOSAJE}

En el caso de los equipos con electrodos activos y pasivos separados, se debe colocar el pasivo preferentemente contraplanar al área de tratamiento, lo más próximo posible, sobre una zona con generosa cantidad de tejido blando sin eminencias oseas. Si el dispersivo es metálico no necesitara de una sustancia de acople, si es de goma con carbono, es probable que si necesite, pero de todas formas deben seguirse las indicaciones del fabricante de cada equipo en particular. Se debe controlar que el mismo no incremente su temperatura. Durante la aplicación se debe regular la potencia del equipo y realizar movimientos en áreas pequeñas de 2 a 3 veces el tamaño del electrodo activo hasta alcanzar la sensación térmica deseada, consiguiendo siempre una hiperemia (3).

Para la regulación de la temperatura se emplean termómetros de infrarrojos y además se tiene en cuenta la escala subjetiva del calor (6):

- G1: imperceptible.

- G2: suave, ligeramente perceptible.

- G3: moderado-fuerte pero no desagradable

- G4: intenso, rosando el umbral del dolor.

- G5: quemante, supera el umbral del dolor.

\section{FUNDAMENTALES EFECTOS FISIOLÓGICOS DE LA RADIOFRECUENCIA CAPACITIVA Y RESIS- TIVA}

Los principales efectos son (1, 7-9):

- Elevación de la temperatura en la profundidad los tejidos.

- Analgesia tanto por elevación del umbral del dolor debido a la mayor presencia endorfinas como al bloqueo de la sensación por el aumento de temperatura.

- Vasodilatación y por tanto aumento del flujo sanguíneo sobre el territorio tratado.

- Efecto relajante.

- Efecto antiinflamatorio tanto por el aumento del drenaje como por el efecto trófico.

\section{Contraindicaciones}

Siguiendo la premisa primun non nocere, se hace necesario considerar las siguientes contraindicaciones para el uso de radiofrecuencia (1).

- Absolutas: marcapasos cardiacos, cáncer/metástasis, artritis, embarazo.

- Relativas: varices/flebitis/tromboflebitis, aplicación sobre glándulas endocrinas y exocrinas, trastorno de sensibilidad, material de osteosíntesis, endoprótesis, infecciones, pacientes que estén ingiriendo vasodilatadores o anticoagulantes. 
Estas contraindicaciones deberán ser evaluadas y decididas en trabajo coordinado entre médico y kinesiólogo, con responsabilidad de informar al paciente.

\section{CONFLICTOS DE INTERÉS Y FUENTE DE FINANCIACIÓN}

El autor declara no poseer conflictos de interés. Fuente de financiación: ninguna.

\section{REFERENCIAS BIBLIOGRÁFICAS}

1. Barrios Marco F [Internet]. Efisioterapia; c2015 [cited 14 Nov 2017]. Electroterapia de alta frecuencia. Diatermia capacitiva y resistiva [approx. 4 screens]. Available from: https://www.efisioterapia.net/articulos/electroterapia-alta-frecuencia-diatermia-capacitiva-y-resistiva

2. Son BC, Kim HS, Kim IS, Yang SH, Lee SW. Percutaneous radiofrequency thermocoagulation under fluoroscopic image-guidance for idiopathic trigeminal neuralgia. J Korean Neurosurg Soc. 2011;50(5):446-452. https://doi.org/10.3340/jkns.2011.50.5.446

3. Ronzio O [Internet]. Agentes físicos; c2008 [cited 14 Nov 2017]. Radiofrecuencia hoy [approx. 6 screens]. Available from: http://www.patriciafroes.com.br/gestao/img/publicacoes/radiofrecuenciahoy.pdf

4. Misaggi B, Gallazzi M, Colombo M, Ferraro M. Articular facets syndrome: diagnostic grading and treatment options. Eur Spine J. 2009;18(Suppl 1):49-51. https://doi.org/10.1007/s00586-009-0987-8

5. Soloman M, Mekhail MN, Mekhail N. Radiofrequency Treatment in Chronic Pain. Expert Rev Neurother. 2010;10(3):469-474. https://doi.org/10.1586/ern.09.153

6. Martínez Morillo M, Pastor Vega JM, Sendra Portero F. Manual de Medicina Física. 1a ed. Madrid: Harcourt Brace; 1998.

7. Shah SGS, Farrow A. Trends in the availability and usage of electrophysical agents in physiotherapy practices from 1990 to 2010: a review. Phys Ther Rev. 2012;17(4):207226. https://doi.org/10.1179/1743288X12Y.0000000007

8. Adair ER, Black DR. Thermoregulatory responses to RF energy absorption. Bioelectromagnetics. 2003;Suppl 6:S17-S38. https://doi.org/10.1002/bem.10133

9. Swicord ML, Balzano $Q$, Sheppard AR. A review of physical mechanisms of radiofrequency interaction with biological systems. 2010 Asia-Pacific International Symposium on Electromagnetic Compatibility, 2010 Apr 12-16; Beijing. IEEE; 2010. p. 21-24. 